\title{
EL POTENCIAL EMPRENDEDOR EN UN PROGRAMA DE EDUCACIÓN EMPRENDEDORA EN LA ESO \\ UN ESTUDIO DE CASO
}

\author{
ENTREPRENEURIAL POTENTIAL IN AN ENTREPRENEURSHIP \\ EDUCATION PROGRAMME IN SECONDARY SCHOOL
}

A CASE STUDY

\begin{abstract}
Antonio Bernal-Guerrero ${ }^{a}$,Antonio Ramón Cárdenas-Gutiérrez ${ }^{a *}$, Ana María Dominguez-Quintero ${ }^{a}$ y Elisabet Montoro-Fernández ${ }^{a}$
\end{abstract}

Fechas de recepción y aceptación: 2 de julio de 2021 y 11 de septiembre de 2021

DOI: https://doi.org/10.46583/edetania_2021.60.929

Resumen: La educación emprendedora se implementa progresivamente en las etapas de educación obligatoria a través de distintos programas y experiencias relacionadas con la configuración de la Competencia en el Sentido de la Iniciativa y el Espíritu Emprendedor. Con la finalidad de transformar las ideas en actos, se intenta formar al alumnado en un conjunto de conocimientos, habilidades y actitudes relacionados con el desempeño empresarial. El potencial emprendedor, comprendido como el conjunto de capacidades y disposiciones para la acción de emprender, será el objeto de estudio. Así pues, esta investigación se centra en conocer cómo las metodologías activas desplegadas en la educación emprendedora inciden, positivamente o no, sobre el potencial emprendedor de los alumnos partícipes en un proyecto de educación emprendedora en $4 .^{\circ}$ de la ESO. El diseño de investigación es mixto, donde la prioridad metodológica corresponderá a la fase cualitativa, que se implementará mediante un estudio de caso centrado en el alumnado participante en el programa de educación emprendedora. La muestra ha sido seleccionada de forma intencional y por conveniencia, según determinados criterios de investigación, tales como accesibilidad al campo de estudio, disponibilidad de los participantes y presencialidad de los investigadores. Las técnicas de recogida de información son el test ATE-S para seleccionar a los participantes y la entrevista en profundidad. El análisis

${ }^{a}$ Facultad de Ciencias de la Educación. Universidad de Sevilla.

* Correspondencia: Universidad de Sevilla. Facultad de Ciencias de la Educación. Calle Pirotecnia, s/n. 41013 Sevilla. España.

E-mail: acardenas1@us.es 
de los resultados nos indica, por un lado, que el desarrollo del potencial emprendedor se encuentra relacionado con metodologías activas de aprendizaje y, por otro lado, que el potencial emprendedor está configurado por indicadores personales.

Palabras clave: potencial emprendedor, educación secundaria obligatoria, educación emprendedora, programas de formación, competencia emprendedora.

Abstract: Entrepreneurial education is progressively implemented in the stages of compulsory education through different programmes and experiences related to the configuration of the Sense of Initiative and Entrepreneurship Competence. In order to transform ideas into actions, the aim is to train students in a set of knowledge, skills and attitudes related to entrepreneurial performance. The entrepreneurial potential understood as the set of capacities and dispositions for entrepreneurial action will be the object of study. Therefore, this research focuses on knowing how the active methodologies deployed in entrepreneurial education affect, positively or not, the entrepreneurial potential of the students participating in an entrepreneurial education project in 4th year of ESO. The research design is mixed, in which the methodological priority will correspond to the qualitative phase, which will be implemented through a case study focused on the students participating in the entrepreneurial education programme. The sample was intentionally and conveniently selected, according to certain research criteria, such as accessibility to the field of study, availability of the participants and presence of the researchers. The data collection techniques used were the ATE-S test to select the participants and the in-depth interview. The analysis of the results indicates, on the one hand, that the development of entrepreneurial potential is related to active learning methodologies, and on the other hand, that entrepreneurial potential is configured by personal indicators.

Keywords: entrepreneurial potential, Compulsory Secondary Education, entrepreneurial education, training programme, entrepreneurial competence.

\section{FundAMENTACIÓN}

Desde hace años, las directrices de la Unión Europa proponen potenciar el emprendimiento desde los primeros años de la escolaridad hasta la Universidad (Sánchez-Torné, Pérez-Suárez, García-Río y Baena-Luna, 2021). La legislación educativa, tanto a nivel nacional como autonómico, ha favorecido, de un modo u otro, su incorporación al sistema educativo. Existe un claro consenso sobre la necesidad de incorporar la educación emprendedora en los diferentes niveles de enseñanza (LOMLOE, 2020). No es de extrañar el conjunto de acciones desplegadas que intenta potenciar el emprendimiento y el espíritu empresarial en la escuela (Azqueta, 2019). En general, las experiencias educativas llevadas a cabo en los niveles inferiores y relacionadas con el emprendimiento se 
caracterizan por el desarrollo de proyectos de creación empresarial o la incorporación de asignaturas optativas u obligatorias vinculadas a la iniciación empresarial, ya sea en la ESO (Iniciación a la actividad emprendedora y empresarial) o en la FP (Empresa e iniciativa emprendedora). Así, se han puesto en práctica múltiples proyectos formativos vinculados al emprendimiento con una idiosincrasia particular en función de su origen, finalidad y localización geográfica. Los programas de emprendimiento han favorecido un escaso desarrollo del dominio personal entendido como la capacidad de poseer iniciativa y autonomía personal.

Una mirada a lo largo y ancho de nuestro contexto social nos hace comprender los múltiples y diversos casos de iniciativa personal que acontecen continuamente (Lisbona y Frese, 2012). El ser humano, por unos u otros motivos, es activo por naturaleza. Construir y desarrollar el futuro personal es una cuestión casi ineludible, más aún en esta época abierta, plural y flexible que nos ha tocado vivir. La configuración identitaria se realiza desde los alegatos de la teoría narrativa de la identidad personal, en busca de un sentido biográfico que facilita la construcción coherente de un relato vital (Linde, 1993). Alcanzar este grado de coherencia narrativa sobre la identidad emprendedora implica sobrepasar la acción de emprender como el cúmulo de habilidades y conocimientos a disposición de una actuación emprendedora concreta. Así, el compendio de definiciones y caracterizaciones de la educación emprendedora parece, más bien, un collage sin una estructura coherente y arraigada en el desarrollo personal. En este sentido, sería conveniente que la intelección de la competencia emprendedora se vinculara más acertadamente con la identidad personal (Liñán, Ceresia y Bernal, 2018; Nielsen y Lassen, 2012).

En la identidad emprendedora se incluye el potencial emprendedor, esto es, el conjunto de capacidades y disposiciones para la acción de emprender, la cual no se minimiza a un simple proceso conductual. Poseer potencial emprendedor no significa que el sujeto tome la decisión de emprender, es decir, no hay una relación causal directa entre intención y potencial emprendedor (Bernal, 2021). Con otras palabras, se puede tener un excelente grado de potencial emprendedor y no acometer ninguna actividad. Por el contrario, puede suceder que se inicien determinadas actividades y se posea un bajo nivel de potencial emprendedor. De este modo, todo nos induce a pensar que las personas con un nivel bajo de potencial emprendedor, aunque tengan una alta tasa 
de capacidad de activación, poseen menos recursos personales para iniciar y mantener una actividad, en contraste con los sujetos con buenos niveles de potencial emprendedor. Un ejemplo sería las mayores tasas de sostenibilidad emprendedora en actividad empresarial mantenidas por aquellas personas que poseen un mayor nivel educativo.

La educación emprendedora posee diferentes interpretaciones. Pensando en el desarrollo personal nos posicionamos en una "enterprise education pedagogy" (Jones y Iredale, 2010), siendo un enfoque más complejo y holístico que otras consideraciones más vinculadas a la empresarialidad. Educar el potencial emprendedor desde una "enterprise education pedagogy" significa orientarnos a la libertad de las personas para generar sus propios proyectos de vida, adaptándose y actuando en función de las oportunidades y obstáculos que puedan aparecer. Inexorablemente, nos inclinamos hacia la consideración de la dimensión personal como potencial emprendedor. Afrontar los inéditos retos de nuestra época y generar, a su vez, proyectos personales, implica el despliegue de un conjunto de acciones por parte del sujeto, en el cual subyace el potencial emprendedor.

Concretamente, en el ámbito de la educación emprendedora se ha indagado en los sustentos del potencial emprendedor desde distintos posicionamientos (Ajzen, 2011; Krueger y Carsrud, 1993; Liñán, 2008; Raab, Stedham, y Neuner, 2005; Santos, Caetano, y Curral, 2014). En nuestro caso, nos centraremos en la perspectiva de Athayde (2009), que comprende el potencial emprendedor como el conjunto de capacidades y disposiciones para la acción de emprender, aunando cinco actitudes susceptibles de aprendizaje y desarrollo mediante la experiencia educativa (Cárdenas y Montoro, 2017, p. 568), a saber:

- Creatividad: capacidad de generar ideas que facilitan el proceso de innovación.

- Liderazgo: capacidad de construir equipos de trabajo, tomar decisiones, negociar y planificar.

- Logro: capacidad de perseverancia y de proactividad hacia la consecución de metas.

- Control personal: capacidad para manejar y autorregular los comportamientos propios. 
- Resolución de problemas: capacidad para afrontar circunstancias de incertidumbre e intensidad.

La práctica educativa relacionada con el desarrollo de la educación emprendedora y el potencial emprendedor, en el sentido que venimos exponiendo, precisa de unas metodologías que favorezcan la construcción de la identidad emprendedora, ya que no es una práctica pedagógica conveniente sostener el desarrollo de la inteligencia emprendedora en el enfoque tradicional de "magister dixit". Ya Sousa (1995) nos indicaba, a finales del siglo pasado, los niveles de retención de los contenidos en función del tipo de práctica educativa que se implemente. Las demandas requeridas a las futuras generaciones precisan transcender los métodos tradicionales de enseñanza y aprendizaje. Así pues, parece necesaria una nueva perspectiva que otorgue mayor relevancia y protagonismo al alumnado. Entendiendo el emprendimiento como un proceso de construcción de proyectos vitales, donde la imaginación y la invención cobran un especial protagonismo, sería conveniente desarrollar una educación emprendedora activa y constructivista, en la que el alumno se convierta en el protagonista de su propio aprendizaje. La concreción de la educación emprendedora significaría implementar un conjunto de estrategias pedagógicas que facilite la construcción y el desarrollo dentro de las prácticas educativas de los indicadores personales de la competencia emprendedora. En este sentido, se debería diseñar e implementar un entramado de métodos educativos que facilitaran la libertad de actuación y la responsabilidad de su aprendizaje, entre otras: aprendizaje basado en proyectos, aprendizaje basado en problemas o aprendizaje cooperativo.

\section{Problema y objetivo de la investigación}

La educación emprendedora es un concepto poliédrico con múltiples opciones de estudio que, sobre todo, ha sido foco de interés a nivel universitario. El informe REDiE (2015) hace patente la carestía de investigaciones en las etapas educativas inferiores respecto a la educación emprendedora. Aunque paulatinamente van surgiendo estudios relacionados con los niveles de educación obligatoria, no se ha estructurado un corpus de conocimiento sólido 
que refleje el estado de la cuestión (Bernal, Cárdenas y Martín, 2021). Hace más de veinte años de la consabida Estrategia de Lisboa, con la que se inició el fomento de la cultura emprendedora en Europa. A pesar del tiempo transcurrido y del desarrollo legislativo (LOCE 2002 y LOE 2006; LOMCE 2015) a favor de la incorporación del emprendimiento dentro del sistema educativo, su impacto y desarrollo no han sido los esperados.

El fomento del emprendimiento ha estado relacionado de forma consistente con el desarrollo empresarial y el autoempleo. No en vano, las políticas relacionadas con la expansión de la cultura emprendedora están imbuidas de esta impronta. El sistema educativo no es ajeno a esto, en el currículum escolar se reflejan nítidamente estas orientaciones empresariales, sin obviar los beneficios que pudieran derivarse de este enfoque de la educación emprendedora para el mundo laboral y las futuras perspectivas profesionales del alumnado. En esta investigación, nuestro interés se dirige hacia el ámbito personal del emprendimiento, centrándonos en el potencial emprendedor del alumnado.

Entendemos que la comprensión de las vivencias escolares del alumnado en el desarrollo de un proyecto de educación emprendedora nos facilitará una visión más profunda de cómo se configura el potencial emprendedor. Ofrecer la palabra al alumnado, tal vez, nos permitirá indagar en los procesos de enseñanza y aprendizaje favorecedores del potencial emprendedor. Con la clara finalidad de comprender cómo se forma el potencial emprendedor en el alumnado definimos el siguiente problema de investigación: conocer y entender cómo las metodologías activas desplegadas en la educación emprendedora inciden, positivamente o no, en el potencial emprendedor de los alumnos partícipes en un proyecto de educación emprendedora dentro de la asignatura Iniciación a la actividad emprendedora y empresarial en $4 .^{\circ}$ de la ESO. A este respecto, el objetivo será analizar qué tipo de metodologías favorecedoras del potencial emprendedor se implementan. A su vez, a partir de las narraciones también intentaremos conocer si se ha incidido de igual forma en todos los dominios del potencial emprendedor o se ha menoscabado alguno. De esta forma, desde la perspectiva del estudiante procuraremos averiguar cómo afectan las metodologías a su potencial emprendedor. 


\section{Metodología}

\subsection{Diseño de la investigación}

Este estudio se caracteriza por ser una investigación mixta, configurada por métodos cuantitativos y cualitativos (Bericat, 1998). El estudio se implementó mediante dos fases con una secuenciación cronológica cuantitativa y cualitativa. La priorización metodológica recaló en la fase cualitativa, siendo clave para la recopilación de los datos, ya que la fase cuantitativa fue utilizada para la selección de los sujetos en función de las puntuaciones obtenidas en el test ATE-S. De este modo, resultó el siguiente diseño de investigación mixta: qt $\rightarrow$ QL.

Específicamente, por una parte, la fase cuantitativa consistió en un diseño cuasiexperimental pretest-postest sin grupo control (Buendía, Colás y Hernández, 1998) y, por otra parte, la fase cualitativa se caracterizó por ser un estudio de casos múltiples (Yin, 2009), en la que los participantes fueron seleccionados en función de las puntuaciones obtenidas en el test ATE-S.

La calidad del estudio de caso se aseguró mediante los criterios que se muestran en la tabla 1 (Gibbs, 2012).

TABLA 1

Criterios y procesos para garantizar la calidad de la investigación

\begin{tabular}{|l|l|}
\hline \multicolumn{1}{|c|}{ Criterios } & \multicolumn{1}{c|}{ Procesos } \\
\hline Credibilidad & La presencia prolongada en el campo de estudio de una investigadora. \\
\hline Transferibilidad & $\begin{array}{l}\text { La muestra fue seleccionada intencionalmente con recopilación de información } \\
\text { de forma profusa y detallada. }\end{array}$ \\
\hline Dependencia & Caracterización exhaustiva de los participantes. \\
\hline Confirmabilidad & $\begin{array}{l}\text { Recogida y procesamiento de la información de forma automática a través del } \\
\text { software Nvivo 11 y contraste de los datos con los participantes. }\end{array}$ \\
\hline
\end{tabular}

Fuente: elaboración propia 


\subsection{Muestra}

Los participantes se seleccionaron con un muestreo intencional (Ruiz, 2009). Los principios aplicados en la selección de los participantes fueron dos: la accesibilidad al contexto de la investigación y los resultados obtenidos en el test ATE-S. Desde la perspectiva cuantitativa, la muestra estaba configurada por 23 alumnos de $4 .^{\circ}$ de la ESO matriculados en la asignatura Iniciación a la actividad emprendedora y empresarial y que participaban en un proyecto de educación emprendedora en el curso 2019/20. Este proyecto de educación emprendedora es una creación propia por parte del equipo docente a partir de su experiencia en la gestión de proyectos emprendedores en alumnado de la ESO. Sin embargo, hubo una reducción de dos sujetos, ya que por motivos de confinamiento por la COVID-19 no pudieron cumplimentar los postest. De este modo, la muestra final se compuso de 21 sujetos. De estos, un $61 \%$ eran alumnas y un $39 \%$ alumnos con edad media de 15,6 años. En la fase cualitativa, de esos 21 sujetos se escogieron a los 5 alumnos que más aumentaron su potencial emprendedor según el test ATE-S. Para garantizar los aspectos éticos de la investigación, se garantizó el anonimato de la muestra y los participantes en la investigación cualitativa fueron identificados mediante el código $\mathrm{Pn}^{\circ} \mathrm{A} / \mathrm{O}$, donde P: identificación del participante con las letras desde la A hasta la E; $n^{\circ}$ : orden de puntuación obtenida en el ATE-S, y A/O: alumno o alumna.

\subsection{Instrumentos}

En la primera fase cuantitativa, los datos se recopilaron con el instrumento ATE-S “Test de Potencial Emprendedor” adaptado al español y con altas cotas de fiabilidad y validez (Bernal, Cárdenas y Athayde, 2021). Este test evalúa el potencial emprendedor de los adolescentes participantes en proyectos de educación emprendedora. La versión española está configurada por una estructura con cinco factores (creatividad, control personal, orientación al logro, liderazgo e intuición para la resolución de problemas) y veintidós ítems en una escala Likert con puntuaciones entre $0=$ completamente en desacuerdo y $5=$ completamente de acuerdo. 
En la segunda fase cualitativa, se utilizó como instrumento de recogida de datos una adaptación de la entrevista en profundidad elaborada en una investigación previa (Cárdenas y Montoro, 2017). Esta adaptación consistió en reestructurar la entrevista inicial relacionada con los cinco factores constitutivos del ATE-S, añadiéndole una dimensión relacionada con las metodologías activas del aprendizaje.

\subsection{Procesos de recogida de información}

Cronológicamente, la recogida de información ha sido desarrollada mediante tres fases:

1. Fase informativa. En esta primera etapa el equipo de investigación mantuvo contacto con un centro educativo, en el que se impartía la asignatura Iniciación a la actividad emprendedora y empresarial, implementada mediante un proyecto de educación emprendedora. Una vez que se informó de la finalidad y los procesos del estudio de campo a la dirección del centro y a la profesora de la asignatura, estos procedieron a confirmar su colaboración en la investigación. A continuación, se informó al alumnado de los objetivos y procesos de la investigación, explicándoles que su participación era voluntaria y se garantizaría el anonimato de sus datos. El alumnado firmó un consentimiento escrito de su participación en la investigación.

2. Fase cuantitativa de recogida de información. Esta fase se dividió en dos momentos; por un lado, la evaluación inicial (pretest) antes de iniciarse el proyecto de educación emprendedora y, por otro lado, la evaluación final (postest) cuando se terminó el proyecto de educación emprendedora. En ambos estadios se utilizó el test ATE-S para conocer el grado de adquisición del potencial emprendedor del alumnado. Finalmente, se seleccionaron a los 5 alumnos con mayor potencial emprendedor.

3. Fase cualitativa de recogida de información. En esta fase se produjo un solo momento de recogida de datos tres semanas después de haber finalizado el proyecto de educación emprendedora. La recogida de información se realizó dentro del horario escolar en la sala de profesores 
habilitada al efecto para la realización de las entrevistas. El conjunto de entrevistas se realizó durante el mismo día con una duración aproximada de unos sesenta minutos respectivamente.

\subsection{Análisis de datos}

El procedimiento de recogida y análisis de los datos ha seguido la secuencia del diseño de investigación mixto planteado $(\mathrm{qt} \rightarrow \mathrm{QL})$. Primero, en la fase cuantitativa (qt) seleccionaron a aquellos alumnos que han logrado aumentar su potencial emprendedor mediante su participación en el proyecto de educación emprendedora. Para ello, se obtuvieron los resultados del alumnado en el test ATE-S mediante las puntuaciones directas alcanzadas del pre y postest y que evidenciaban un cambio favorable en el potencial emprendedor del alumnado. Segundo, en la fase cualitativa (QL) se implementó un análisis del contenido de las entrevistas realizadas a los cinco participantes. Este proceso de análisis cualitativo de los datos se implementó a través de la transcripción de las entrevistas, codificación de los datos, reducción de estos y emergencia del árbol de categorías (tabla 2).

TABLA 2

Árbol de categorías

\begin{tabular}{|l|l|}
\hline \multicolumn{2}{|c|}{ Árbol de categorías } \\
\hline \multicolumn{1}{|c|}{ Categorias } & \multicolumn{1}{c|}{ Subcategorías } \\
\hline \multirow{4}{*}{ Métodos activos de aprendizaje } & Aprendizaje basado en proyectos \\
\cline { 2 - 3 } & Aprendizaje basado en Problemas \\
\cline { 2 - 3 } & Aprendizaje cooperativo \\
\hline \multirow{5}{*}{ Indicadores Personales } & Creatividad \\
\cline { 2 - 2 } & Liderazgo \\
\cline { 2 - 2 } & Orientación al logro \\
\cline { 2 - 2 } & Control personal \\
\cline { 2 - 2 } & Resolución de problemas \\
\hline
\end{tabular}

Fuente: elaboración propia 


\section{Resultados}

\subsection{Métodos activos de aprendizaje}

\subsubsection{Aprendizaje basado en proyectos}

El conjunto de entrevistas nos sugiere que el aprendizaje basado en proyectos es la metodología principal que se ha utilizado en el desarrollo de este proyecto de educación emprendedora.

A1A: "La asignatura se desarrolla mediante un proyecto que hacemos durante todo el curso. La realización del proyecto es lo más importante de todo".

El alumnado narra que la realización del proyecto se ejecuta a través de diferentes fases con una duración determinada. Estas fases poseen cierto grado de dificultad. Según comentan los entrevistados, no es un proceso de consecución fácil alcanzable sin esfuerzos individuales o grupales por parte del alumnado.

C2O: "Al principio creemos que en los proyectos se trata hacer trabajitos y ya está (...) hacer proyectos es algo muy complejo que necesita mucha atención de nosotros (...) te despistas un poco o dejas pasar algo de tiempo y las cosas se complican bastante".

Además, consideran que el aprendizaje basado en proyectos es una metodología adecuada para adquirir aprendizajes relacionados con los conocimientos, habilidades y actitudes en la gestión de proyectos (García, Villaverde, Benito y Muñoz, 2020). Sin embargo, también plantean que, en ocasiones, se han encontrado desorientados y han precisado la guía y orientación de la profesora, ya que se encontraban colapsados ante la abrumadora cantidad de tareas y actividades que debían realizar.

D4A: "Con esta forma de dar clase se aprende mucho sobre cómo hacer las cosas, tienes que aplicar los conocimientos que aprendes, es una metodología muy útil (...) te hacer ver hasta qué punto dominas los conocimientos y qué lagunas tienes. Muchas veces, te crees que sabes el contenido y te das cuenta de que te falta por aprender mucho contenido. Gracias, que siempre estaba la profesora ayudándonos". 


\subsubsection{Aprendizaje basado en problemas}

Todos los informantes coinciden en que en la ejecución del proyecto de educación emprendedora surgen diferentes problemas. A este respecto, describen que emergen situaciones problemáticas en el desarrollo del proyecto. Estos problemas están relacionados con diferentes procesos relacionados con la gestión, los contenidos, la temporalización de los plazos marcados, la aplicabilidad o la resolución de conflictos en la realización de las tareas y actividades (Román, 2021).

B5O: "En el proyecto siempre surgen problemas, no habiamos casi resuelto uno, cuando ya veíamos en el horizonte el siguiente problema".

E3A: "Cuando hacíamos el proyecto han aparecido distintos problemas. Algunos días, estábamos saturados de actividades. A veces, no habíamos planificado bien las tareas o actividades. Otras no estábamos de acuerdo en cómo haciamos las actividades y nos peleábamos".

Paulatinamente, frente a estas situaciones los informantes describen que la profesora les ha ido enseñando cómo afrontar y gestionar los problemas. Las narraciones nos indican que la profesora ha implementado el proyecto basándose en el aprendizaje de problemas (Freire, 2021).

A1A: "Al inicio del proyecto todo iba muy bien, pero poco a poco, empezaron los problemas, entonces la profesora empezó a enseñarnos cómo resolver estos problemas".

B5O: "La profesora nos explicó durante varias clases sobre cómo debemos resolver los problemas. Hay una forma, un método con sus fases para poder gestionar los problemas. No siempre nos ha funcionado, pero hemos conseguido aplicar ese método para resolver los problemas que nos aparecian en la gestión del proyecto".

A este respecto, los alumnados expresan que es necesario y conveniente aprender a resolver problemas de manera metódica, pues de una forma razonada y nada azarosa logran minimizar el impacto negativo de los problemas sobre la gestión del proyecto que estaban ejecutando (Ayala, 2020). 
E3A: "No sabía que se podía aprender a resolver problemas. Parecía que no, pero si aplicamos las fases y los pasos que nos han explicado podemos reducir los problemas, aunque no es fácil de hacer y tenemos que seguir aprendiendo. A mí, todo esto me ha ayudado para finalizar el proyecto".

\subsubsection{Aprendizaje cooperativo}

La realización del aprendizaje basado en proyectos y en problemas no se ha realizado de forma individual, sino que ha transitado desde un aprendizaje grupal al inicio del proyecto de educación emprendedora hacia un aprendizaje cooperativo a lo largo y final del proyecto.

C2O: "Siempre hemos trabajo en grupo. Al principio formamos grupos de trabajo y cada grupo de trabajo ha realizado su propio proyecto".

E3A: "Cuando empezamos las actividades lo hacíamos de forma grupal, pero nos dimos cuenta de que no solo era un trabajo en grupo, sino que todos dependiamos de todos para llegar al final del proyecto. Vamos, que teníamos que cooperar unos con otros, que sin mi compañero yo sólo no podía hacer nada y al revés".

La apreciación del aprendizaje cooperativo ha sido positiva por parte de los informantes. Valoran que el aprendizaje cooperativo es una forma muy válida para aprender, ya que se sustenta en un aprendizaje mutuo e interdependiente dentro del grupo de trabajo (Aguilera, 2020).

D4A: "Aprender entre todos juntos está muy bien. Uno cree que lo sabe todo y cuando escucha a los compañeros del grupo siempre aprende cosas desde un punto de vista distinto, y entonces te dices, yo esto no lo había pensado así. Creo que aprender entre todos los compañeros nos enriquece lo que aprendemos en las clases".

Aunque se reconoce los beneficios del aprendizaje cooperativo, no es menos cierto que las narraciones nos indican que este tipo de aprendizaje precisa de una guía u orientación por parte del profesorado (Guzmán y Montenegro, 
2020). No se trata únicamente de compartir unos espacios y tiempos educativos comunes.

A1A: "Cuando hemos trabajado con el grupo hemos necesitado que la profesora nos indicara cómo debiamos cooperar, sobre todo al principio del proyecto, pero después todo se fue haciendo más fácil y lográbamos hacer las tareas juntas, pero la profesora siempre estaba mirando cómo estaba el grupo de trabajo".

\subsection{Indicadores personales}

\subsubsection{Creatividad}

La generación del proyecto de emprendimiento ha supuesto el inicio de procesos creativos por parte del alumnado. Las actividades, tareas y producto final se han caracterizado por poseer altas de dosis de creatividad por parte de todos los grupos de trabajo.

C2O: "Aqui es imposible aburrirse, siempre salian cosas nuevas. Nos hemos tenidos que inventar muchas cosas en las fases del proyecto, aunque buscábamos en internet la información, al final teníamos que crear algo con la información que teníamos".

Si bien la profesora ha orientado los procesos creativos, han sido los propios alumnos quienes han propuesto sus ideas de proyecto y las fórmulas para poder conseguir el producto final planteado.

B5O: "Nuestra profe nos daba alguna pista, pero éramos nosotros quienes teníamos que generar todo. La idea de nuestro proyecto las creamos nosotros, claro está, ella nos ayudó a perfeccionarla, pero nunca nos dijo esta idea es la que tenéis que hacer en el proyecto".

El desarrollo creativo generado no ha sido puntual y concreto en el tiempo, sino que se gestionaron procesos de planificación a lo largo del tiempo para la realización y materialización de las ideas generadas (Ricci, 2020). 
E3A: "Cuando nos venía una idea, después había que estudiar si se podía realizar, y si se podia realizar, nuestro grupo se ponía a describir todos los pasos que teníamos que hacer para hacerla realidad".

D4A: "La idea final de proyecto la desarrollamos durante todo el curso y pasamos por todas las fases. Esto no es tengo una idea y ya está, sino que tenemos que hacerla realidad".

Según comentan los participantes, el proceso creativo ha significado un proceso de reflexión constante, planteándose cuestiones claves sobre el origen, desarrollo, planificación y producción de la idea de proyecto (Elisondo y Piga, 2020). De este modo, todo indica que se ha generado un cierto sentido crítico sobre la ejecución del proyecto.

D4A: "En el proyecto no dábamos nada por hecho. Pensábamos sobre cómo mejorarlo, cómo ajustar las acciones y tareas, cómo planificar. No era un corta y pega, sino que habia que darle muchas vueltas a la cabeza".

\subsubsection{Liderazgo}

Las narraciones indican la espontaneidad inicial en el desarrollo del liderazgo, esto es, los alumnos con más propensión hacia el liderazgo ejercieron ese rol, mientras que otros alumnos no eran tan proclives hacia las tareas de liderazgo.

B5O: "Al principio yo no le decía a la gente lo que tenía que hacer. Cuando empezamos con el grupo de trabajo mi amiga Ana fue quien empezó a decir qué hacíamos y cómo lo hacíamos".

Sin embargo, los alumnos entrevistados explican que durante el proyecto la profesora fue asignando roles a los distintos miembros de los equipos de trabajo, donde tenían que ejercer un rol de liderazgo del equipo de trabajo. En cierto modo, la práctica educativa de la profesora ha distribuido durante el proyecto el rol de líder para que todos los alumnos tuvieran la oportunidad de desarrollar cierta capacidad de liderazgo. 
C2O: "A mi no me gusta dirigir, pero durante el proyecto he tenido que dirigir, porque la profesora me daba tareas en las cuales yo tenía que organizar con mis compañeros de grupo. Me costó algo de trabajo porque a mí no me gusta mucho y prefiero pasar desapercibida, pero al final logré hacerlo".

El alumnado reconoce que ha adquirido o mejorado las habilidades sociales y comunicativas, puesto que ha tenido que negociar sus planteamientos y expresar sus sentimientos o posiciones frente al resto de compañeros de grupo (Oliver, Arenal, Armuña, García, Álvarez y Oviedo, 2021). Además, afirman que ejercer el liderazgo durante determinadas actividades les ha facilitado el desarrollo de la empatía (Tapia, López y Rodríguez, 2021).

E3A: "Nosotros hemos tenido que hablar mucho para realizar todo el proyecto. A veces, hemos llegado a acuerdos, después de discutir sobre qué hacíamos. Antes no pensaba en la forma de pensar de los demás. Ahora intento ponerme en lugar de mi compañero para comprender qué me quiere decir".

\subsubsection{Orientación al logro}

Los participantes vienen a referirse que, tanto ellos como el resto de los compañeros del curso, han estado motivados para la realización del proyecto de educación emprendedora. Entre los razonamientos explicados se encuentra la realización de una asignatura de forma diferente, mayores posibilidades de actuación por su parte y ver cómo pueden alcanzar determinados objetivos planteados por el equipo de trabajo.

A1A: "Todos queríamos hacer el proyecto, ya que era hacer algo diferente al resto de asignaturas. En este proyecto nos han dejado hacer por nosotros mismos, no teníamos a la profesora toda la clase encima de nosotros. Además, nos planteábamos cosas para poder conseguirlas".

Aunque existe una percepción de alta motivación, no es menos cierto que también han tenido episodios de baja motivación, sobre todo, cuando se han presentado dificultades de realización en alguna de las fases del proyecto (Barriopedro, Quintana y Ruiz, 2018). En estos momentos, reconocen que han 
necesitado la orientación de la profesora y que han sentido deseos de abandonar el proyecto.

B5O: "A veces se nos quitaban las ganas de todo. Habia días en que todo salía mal y entraba ganas de decir hasta aqui hemos llegado, pero siempre estaban ahí los compañeros para ayudarnos, pero lo más importante era que la profesora nos animaba a seguir hacia delante".

La definición de metas focales e instrumentales y su posterior consecución es un excelente indicador del desarrollo de la motivación de logro del alumnado participante. El planteamiento del producto final del proyecto (meta focal) y la realización de diferentes estadios intermedios (metas instrumentales) han permitido que el alumnado construya una estructura de metas que guíe su comportamiento para la consecución de los objetivos definidos (Chan, Domínguez y Santos, 2020).

E3A: "Lo importante ha sido saber qué queríamos conseguir, hacia dónde dirigíamos todos nuestros esfuerzos. Tener una meta al final nos ha permitido saber qué camino debiamos tomar en cada momento. Así, en cada fase del proyecto nos proponíamos conseguir pequeños objetivos".

\subsubsection{Control personal}

La capacidad de autorregular el propio comportamiento y, con ello, la actuación general de cada grupo de trabajo se han constituido como factores necesarios y convenientes en la implementación del proyecto.

D4A: "Hemos aprendido a hacer lo que teníamos que hacer, como digo yo, me planteaba, esto me lleva por buen camino o no, y según la respuesta que daba asi actuaba".

C2O: "Todo el grupo actuaba bien, sabíamos qué queríamos y nos poníamos a ellos". 
Todo ello no ha sido óbice para que no aparecieran comportamientos distorsionadores. Sin embargo, la regulación grupal ha facilitado que los grupos de trabajo fueran dinámicos y consecuentes con los objetivos que se planteaban.

B5O: "Alguna vez algún compañero intentaba no hacer nada, pero el grupo le recriminaba esta actitud y de nuevo se tenía que poner las pilas".

Un ejemplo esclarecedor sobre el control personal es el desarrollo de normas por parte de los propios alumnos con la intención de que todos supieran cómo debían comportarse. La generación de normas de forma autónoma presupone cierto grado de madurez personal y moral, facilitando en buen grado el control y la gestión de la conducta propia y los comportamientos grupales. La creación e implementación de normas de forma autónoma frente a la heteronomía normativa facilita el control y la regulación personal (Santillán, Jaramillo y Santos, 2021).

A1A: "Nadie nos ha dicho cómo nos teníamos que comportar, nosotros mismos nos pusimos nuestras normas. La profesora no decía debes hacer esto o lo otro, sino que al principio ella nos explicó lo importante de comportarnos bien y nosotros mismos fuimos generando algunas reglas para evitar problemas".

\subsubsection{Resolución de problemas}

Es obvio que la gestión de un proyecto implica la aparición de situaciones problemáticas de diversa índole. Si bien se ha implementado un aprendizaje basado en problemas para acometer las dificultades, este no se desarrolla de forma repentina, sino que precisa de cierta periodización que facilite la adquisición de las habilidades y conocimientos necesarios para la gestión de los problemas (Sanabria y Riobueno, 2017). Mientras esto sucede, las circunstancias problemáticas acontecen de una u otra forma y no se detienen. Por ello, es conveniente conocer cómo afrontan los problemas hasta que logran alcanzar cierta capacidad efectiva de resolución de problemas (Travieso y Ortiz, 2018).

Según las narraciones, los problemas se escindirían en individuales y colectivos. Los primeros serían aquellos problemas que han afrontado los alumnos 
por sí mismos y los segundos se definirían como las dificultades grupales en la realización del proyecto.

E3A: "Cuando estaba haciendo las actividades, algunas veces me surgían problemas que intentaba solucionar como podía, aunque no sabía verdaderamente cómo hacerlo, era más bien un ensayo de acierto y error".

D4A: "Me acuerdo de que cuando empezamos a trabajar todo juntos, habia mucho lio, cada uno intentaba hacer lo que podía cuando empezaban los problemas en el desarrollo del proyecto, pero no sabíamos a ciencia cierta qué estábamos haciendo".

Las dificultades principales para alcanzar una buena efectividad, sobre todo, venían de la incapacidad de saber reflexionar adecuadamente o de analizar las diferentes situaciones que aparecían.

C2O: "Al principio intentábamos solucionar los problemas de forma superficial y no lográbamos resolver nada. Al contrario, se complicaba más".

\section{Conclusiones}

Las narraciones nos vienen a indicar que este proyecto se ha implementado mediante metodologías activas de enseñanza y aprendizaje que, a su vez, han incidido positivamente en los dominios del potencial emprendedor. No sin críticas, cada vez más, se están introduciendo estas metodologías denominadas como activas, caracterizadas por la participación y el aprendizaje dinámico del alumnado (Alcalá, Santos y Leiva, 2020). Conscientes de la complejidad de transformar los procesos de enseñanza y aprendizaje anclados en modelos educativos pasivos en relación con el papel que desempeña el alumnado en su propio aprendizaje. La introducción de la educación emprendedora ha supuesto un empuje y una ruptura con los cánones metodológicos más rígidos y reduccionistas, fomentando inéditas líneas metodológicas, como Design Thinking o Lean Canvas, entre otras. 
Proverbialmente, Ken Robinson y Lou Aronica (2015) nos apelaban a comprender la escuela como una institución con claras reminiscencias dieciochescas en línea con las argumentaciones de Fernández Enguita (1990). Sin embargo, la estructura, el funcionamiento y la organización y gestión de las aulas nada tienen que ver con las nuevas demandas exigidas a la escuela. Del mismo modo, metafóricamente, Marina (2016) nos describía la escuela como un diplodocus difícil de despertar frente a los cambios sociales, culturales, económicos y políticos que están aconteciendo. A este respecto, la introducción de la educación emprendedora ha promovido la introducción de metodologías de enseñanza y aprendizaje más activas, rompiendo con los cánones metodológicos más tradicionales.

La metodología implementada en este proyecto de educación emprendedora se caracterizaría por lo que denominaríamos eclecticismo metodológico inclusivo. Así, del total de narraciones se desprende que la metodología central se ha basado en el aprendizaje basado en proyectos, incorporando, a su vez, el aprendizaje basado en problemas y el aprendizaje cooperativo (Meroño, Calderón y Arias, 2021). La información aportada por los participantes nos indica que estas metodologías son convenientes para construir conocimientos y desarrollar las habilidades emprendedoras, pero sobre todo incide en los indicadores personales del potencial emprendedor, tales como la creatividad, el liderazgo, la orientación al logro, el control personal y la resolución de problemas. Inequívocamente, se deduce de las argumentaciones explicadas que el alumnado está a favor de estas metodologías activas (Gómez, Del Pilar, Falcón y Llamas, 2020). Las connotaciones positivas para el desarrollo del potencial emprendedor parecen que son consistentes. Aunque los datos recabados reflejan que la implementación de este eclecticismo metodológico inclusivo no ha estado exenta de dificultades. La consecución del proyecto ha supuesto desarrollar determinados principios, tareas, procedimientos y actividades educativas sin experiencia previa del alumnado al respecto. En su conjunto, el proyecto ha significado una innovación educativa, pero también un reto pedagógico de incalculable valor para la construcción del potencial emprendedor de los participantes.

En relación con esto, de las narraciones se infiere que las tres metodologías activas utilizadas en el proyecto han incidido positivamente sobre el potencial 
emprendedor. Se aprecia que los indicadores personales del emprendimiento: creatividad, liderazgo, orientación al logro, control personal y resolución de problemas, han sido desarrollados con cierto grado de ecuanimidad. En este sentido, el proyecto de educación emprendedora ha contribuido a la mejora de los distintos indicadores del potencial emprendedor. Con una mayor complejidad metodológica, la imbricación de cada uno de los métodos ha permitido contrarrestar, en alguna medida, los obstáculos que han ido apareciendo a lo largo del proyecto. Si bien el rol del profesorado no ha sido objeto de análisis por sí mismo, se interpreta que la docente responsable de la ejecución del proyecto ha sido una figura clave para su buen desarrollo. Así, ha otorgado tiempos y espacios de actuación para el alumnado, donde ella ha ejercido como guía y apoyo educativo reorientando la ejecución del proyecto de educación emprendedora bajo el prisma del eclecticismo metodológico inclusivo.

En general, los datos obtenidos sostienen la premisa de que el potencial emprendedor se ha desarrollado positivamente. Esta investigación ha sido un estudio cualitativo sobre una experiencia puntual no extrapolable que posee sus propias limitaciones, como la selección de la muestra o la falta de datos cuantitativos con mayor robustez estadística. Por esta razón, en futuras líneas de investigación sería conveniente analizar una mayor muestra que haya obtenido una mejora del potencial emprendedor, contrastar la visión de alumnado con alto y bajo potencial emprendedor o conocer el efecto e impacto de estas metodologías sobre el potencial emprendedor.

\section{Agradecimientos}

Este artículo se origina dentro el proyecto "Formación del Potencial Emprendedor. Generación de un Modelo Educativo de Identidad Emprendedora", con referencia PID2019-104408GB-I00, dentro del Plan Estatal 2017-2020 de Proyectos I+D+i (Generación de Conocimiento), financiado por el Ministerio de Ciencia e Innovación - Agencia Estatal de Investigación/10.13039/501100011033. 


\section{BiBLIOGRAFÍA}

Aguilera, M. (2020). El aprendizaje cooperativo y el desarrollo de las habilidades cognitivas. Revista EDUCARE-UPEL-IPB-Segunda Nueva Etapa $2.024(1), 51-74$.

AJZEN, I. (2011). The theory of planned behaviour: Reactions and reflections. Psychology and Health 26(9), 1113-1127.

Alcalá, M. J., Santos, M. J. y Leiva, J. J. (2020). Metodologías activas e innovadoras en la promoción de competencias interculturales e inclusivas en el escenario universitario. European Scientific Journal 16(40), 6-23.

Athayde, R. (2009). Measuring enterprise potential in young people. Entrepreneurship Theory and Practice 33(2), 481-500.

Ayala, J. (2020). El aprendizaje basado en problemas y el desarrollo de las habilidades del pensamiento crítico. Revista EDUCA UMCH 15, 1-17.

AzQueTA, A. (2019). Análisis del concepto “emprendedor" y su incorporación al ámbito emprendedor. Teoría de la Educación 31(1), 57-80.

Barriopedro, M., Quintana, I. y Ruiz, L. (2018). La perseverancia y pasión en la consecución de objetivos: validación española de la Escala Grit de Duckworth. RICYDE. Revista Internacional de Ciencias del Deporte 54(14), 297-308.

Bericat, E. (1998). La integración de los métodos cuantitativo y cualitativo en la investigación social. Barcelona: Ariel.

Bernal, A. (2021). Educación Emprendedora. Fundamentos y elementos para la transferencia e innovación pedagógica. Madrid: Ed. Síntesis.

Bernal, A., CÁrdenas, A. R. y Athayde, R. (2021). Test de potencial emprendedor: adaptación al español (ATE-S). Bordón. Revista de Pedagogía 73(1), 19-37.

Bernal, A. Cárdenas, A. R. y Martín, A. (coords.) (2021). Veinte años de educación emprendedora. Madrid: Octaedro.

Buendía, L., Colás, P. y Hernández, F. (1998). Métodos de Investigación en psicopedagogía. Madrid: McGraw-Hill.

CÁrdenas, A. R. y Montoro, E. (2017). Evaluación de un proyecto de educación emprendedora en la ESO. La visión del alumnado. RIE. Revista de Investigación Educativa 35(2), 563-581. 
Chan, I., Domínguez, N. y Santo, D. (2020). Sentido de vida y establecimiento de metas en estudiantes de bachillerato. ACADEMO. Revista de Investigación en Ciencias Sociales y Humanidades 7(1), 22-32.

Elisondo, R. y PigA, M. (2020). Todos podemos ser creativos. Aportes a la educación. Diálogos sobre educación. Temas actuales en investigación educativa 11(20), 1-22.

EuRYdice ESPAÑA-REDIE (2015). La educación para el emprendimiento en el sistema educativo español. Año 2015. Ministerio de Educación, Cultura y Deporte. España.

FERnÁndez, M. (1990). La cara oculta de la escuela. Madrid. Siglo XXI España Editores.

Freire, E. (2021). El aprendizaje basado en problemas, un reto a la enseñanza superior. Revista Conrado 17(80), 295-303.

García, V., Villaverde, V., Benito, V. y Muñoz, R. (2020). Aprendizaje basado en proyectos y estrategias de evaluación formativas: Percepción de los estudiantes universitarios. Revista Iberoamericana de Evaluación Educativa 13(1), 93-110.

GiBBs, G. (2012). El análisis de datos cualitativos en investigación cualitativa. Madrid: Morata.

Gómez, I., Del Pilar, M., Falcón, I. y Llamas, J. (2020). Adaptación de las Metodologías Activas en la Educación Universitaria en Tiempos de Pandemia. Revista Internacional de Educación para la Justicia Social 9(3), 415-433.

Guzmán, B. y Montenegro, M. (2020). Evaluación de un Programa de capacitación docente para optimizar la Estrategia de Aprendizaje Cooperativo. Controversias y Concurrencias Latinoamericanas 12(21), 323-464.

Jones, B. e IredAle, N. (2010). Enterprise education as pedagogy. Education + Training 52(1), 7-19.

Krueger, N. F. y CARSRUd, A. L. (1993). Entrepreneurial intentions: applying the theory of planned behaviour. Entrepreneurship \& Regional Development 5(4), 315-330.

Ley Orgánica 10/2002, de 23 de diciembre, de Calidad de la Educación. Boletín Oficial del Estado (BOE), 307, 24 de diciembre de 2002.

Ley Orgánica 2/2006, de 3 de mayo, de Educación. Boletín Oficial del Estado (BOE), 106, mayo. 
Ley Orgánica 3/2020, de 29 de diciembre, por la que se modifica la Ley Orgánica 2/2006, de 3 de mayo, de Educación. Boletín Oficial del Estado (BOE), 340, sec. I, 30 de diciembre de 2020.

Linde, C. (1993). Life Stories: The Creation of Coherence. New York: Oxford University Press.

LiÑÁN, F. (2008). Skill and value perceptions: how do they affect entrepreneurial intentions? International Entrepreneurship and Management Journal 4(3), 257-272.

Liñán, F., Ceresia, F. y Bernal, A. (2018). Who Intends to Enroll in Entrepreneurship Education? Entrepreneurial Self-Identity as a Precursor. Entrepreneurship Education and Pedagogy 1(3), 222-242.

Lisbona, A. y Frese, M. (2012). Iniciativa personal. Cómo hacer que las cosas sucedan. Madrid: Pirámide.

Marina, J. A. (2016). Despertad al diplodocus. Una conspiración educativa para transformar la escuela y todo lo demás. Barcelona: Ariel.

Meroño, L., Calderón, A. y Arias, J. L. (2021). Pedagogía digital y aprendizaje cooperativo: efecto sobre los conocimientos tecnológicos y pedagógicos del contenido y el rendimiento académico en formación inicial docente. Revista de Psicodidáctica 26(1), 53-61.

Nielsen, S. y LASSEN, A. (2012). Identity in entrepreneurship effectuation theory: a supplementary framework. International Entrepreneurship and Management Journal 8(3), 373-389.

Oliver, M., Arenal, A., Armuña, C., García, T., Álvarez, J. y Oviedo, J. (2021). Estilos de aprendizaje e inteligencias múltiples para impulsar el emprendimiento y el liderazgo social desde la educación secundaria. AECA: Revista de la Asociación Española de Contabilidad y Administración de Empresas 133, 53-57.

Raab, G., Stedham, Y. y Neuner, M. (2005). Entrepreneurial potential: An exploratory study of business students in the U.S. and Germany. Journal of Business and Management 11(2), 71-88.

Ricci, P. (2020). Una revisión general sobre la creatividad. Revista Científica Arbitrada de la Fundación MenteClara 5, 1-12.

Robinson, K. y ARonicA, L. (2015). La revolución que está transformando la educación. Barcelona: Ediciones Grijalbo. 
RomÁn, F. (2021). La Neurociencia detrás del aprendizaje basado en problemas (ABP). Journal of Neuroeducation 1(2), 50-56.

Ruiz, J. (2009). Metodología de la investigación cualitativa. Bilbao: Universidad de Deusto.

SAnABria, M. y Riobueno, G. (2017). Solucionando dificultades en el aula: una estrategia usando el aprendizaje basado en problemas. Revista Cuidarte 8(3), 1907-1918.

Sánchez-Torné, I., Pérez-Suárez, M., García-Río, E. y Baena-Luna, P. (2021). ¿Cómo influye el género en la intención emprendedora? Un análisis del alumnado universitario. Revista de Investigación Educativa 39(2), 427-444.

SAntillán, J., Jaramillo, E. y SAntos, R. (2021). El aprendizaje cooperativo como nueva metodología en el aula. Polo del Conocimiento 6(1), 10601078.

Santos, S., Caetano, A. y Curral, L. (2014). Psychosocial aspects of entrepreneurial potential. Journal of Small Business and Entrepreneurship 26(6), 661-685.

Souda, D. A. (1995). How the Brain Learns. Reston, Virginia: The National Association of Secondary School Principals, 31-88.

TAPIA, M., López, S. y RodríGuez, I. (2021). El manejo de las emociones, habilidad estratégica en el liderazgo disruptivo. Indiciales 1(1), 1-11.

Travieso, D. y Ortiz, T. (2018). Aprendizaje basado en problemas y enseñanza por proyectos: alternativas diferentes para enseñar. Revista Cubana de Educación Superior 37(1), 124-133.

Y In, R. (2009). Case Study Research: Design and Methods. Thousand Oaks, Estados Unidos: Sage. 